\title{
The Influence of Auxiliary Ligands on the Photophysical Characteristics of a Series of Ruthenium (II) Polypyridyl Complexes
}

Luke O'Neill

Technological University Dublin, Luke.oneill@tudublin.ie

Laura Perdisatt

Technological University, laura.perdisatt@gmail.com

Christine O'Connor

Technological University Dublin, christine.oconnor@tudublin.ie

Follow this and additional works at: https://arrow.tudublin.ie/materart

Part of the Physical Chemistry Commons

\section{Recommended Citation}

O'Neill, L., Perdisatt, L., O'Connor, C. The influence of auxiliary ligands on the photophysical characteristics of a series of ruthenium (II) polypyridyl complexes. Journal of physical chemistry Oct. 2012. doi:10.1021/ jp306927a

This Article is brought to you for free and open access by the Materials Synthesis and Applications at ARROW@TU Dublin. It has been accepted for inclusion in Articles by an authorized administrator of ARROW@TU Dublin. For more information, please contact arrow.admin@tudublin.ie, aisling.coyne@tudublin.ie, gerard.connolly@tudublin.ie.

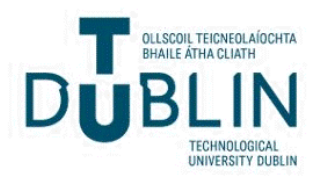




\section{The Influence of Auxiliary Ligands on the Photophysical Characteristics of a Series of Ruthenium (II) Polypyridyl Complexes}

Luke Andrew O'Neill, Laura Perdisatt, and Christine M O'Connor

J. Phys. Chem. A, Just Accepted Manuscript • Publication Date (Web): 11 Oct 2012

Downloaded from http://pubs.acs.org on October 12, 2012

\section{Just Accepted}

"Just Accepted" manuscripts have been peer-reviewed and accepted for publication. They are posted online prior to technical editing, formatting for publication and author proofing. The American Chemical Society provides "Just Accepted" as a free service to the research community to expedite the dissemination of scientific material as soon as possible after acceptance. "Just Accepted" manuscripts appear in full in PDF format accompanied by an HTML abstract. "Just Accepted" manuscripts have been fully peer reviewed, but should not be considered the official version of record. They are accessible to all readers and citable by the Digital Object Identifier (DOI®). "Just Accepted" is an optional service offered to authors. Therefore, the "Just Accepted" Web site may not include all articles that will be published in the journal. After a manuscript is technically edited and formatted, it will be removed from the "Just Accepted" Web site and published as an ASAP article. Note that technical editing may introduce minor changes to the manuscript text and/or graphics which could affect content, and all legal disclaimers and ethical guidelines that apply to the journal pertain. ACS cannot be held responsible for errors or consequences arising from the use of information contained in these "Just Accepted" manuscripts. 


\title{
The Influence of Auxiliary ligands on the Photophysical
}

\section{Characteristics of a series of Ruthenium (II) Polypyridyl}

\section{Complexes}

\author{
Luke O'Neill', , Laura Perdisatt ${ }^{2}$ and Christine O'Connor ${ }^{2}$ \\ ${ }^{1}$ FOCAS Research Institute, Dublin Institute of Technology, Kevin St, Dublin 8, Ireland. \\ ${ }^{2}$ FOCAS Research Institute/School of Chemical and Pharmaceutical Sciences, Dublin Institute of \\ Technology, Kevin St, Dublin 8, Ireland.

\section{Luke.oneill@dit.ie}




\begin{abstract}
A series of ruthenium polypyridyl complexes were studied using UV/Vis absorption and luminescence spectroscopy as well as luminescence lifetime determination by time correlated single photon counting (TCSPC). The complexes were characterised with regard to the variation in the electronic band-gap as a result of the sequential variation of the auxiliary ligand 2,2'-bipyridine (bpy), 1,10-phenanthroline (phen) and 2,2'-biquinoline (biq) ligands while the main ligand remained constant for three different main ligand types. Luminescence yields were calculated and correlated with structural and electronic variation. It was found that both the absorption and emission characteristics could be tailored through the systematic variation of the reduction potential of the individual auxiliary ligand. This was shown to be the case regardless of the functional group at the end of the main ligand. Stokes shift and Raman spectroscopy was employed as a means to gauge the effect of ligand change on the conjugation and vibrational characteristics of the complexes. Luminescence yield and lifetimes were also shown to be well-defined with regards to systematic structure variations. The well-defined trends established elucidate the effect which variation of auxiliary ligands has on the electronic and excited state characteristics of the ruthenium polypyridyl systems. These well-defined relationships can potentially be extended to optimise luminescence yield and lifetimes and therefore suitability of such compounds for the application in for example photodynamic therapy.
\end{abstract}

KEYWORDS: - Ruthenium polypyridyl complexes, Electronic Spectroscopy, Luminescence Lifetimes, Time Correlated Single Photon Counting, 


\section{Introduction}

There has been a major increase in research into and synthesis of inorganic therapeutics due to the limited activity of drugs such as cis-platin and additionally the drug resistance phenomena which lower their impact $[1,2,3]$. The relative success of cis-platin has stimulated the search for cytotoxic compounds with more acceptable toxicity profiles $[4,5]$. Research has gone into ruthenium, titanium and gallium complexes, of which some have gone onto clinical trials phase (I) and (II) $[6,7,8]$. Other potential applications for which ruthenium based complexes are at the forefront of research are concentrators for solar cells, oxygen sensors and photoactive drugs [9, 10, 11].

Primary research focus has been on the therapeutic properties of the complexes. Several different ruthenium (II) and (III) complexes have exhibited good antitumour and anti-metastic properties [12, 13, 14]. These complexes also showed relatively low cytotoxicity as compared to cis-platin. They proved to have a higher therapeutic dose than cis-platin [14]. This is an extremely desirable factor as a drug with a narrow therapeutic range has little difference between toxic and therapeutic dose. A prime example is the $\mathrm{Ru}(\mathrm{III})$ complex commonly known as NAMI-A [1mH][trans $\left.\mathrm{RuCl}_{4}(\mathrm{DMSO}-\mathrm{S})(\mathrm{Im})\right]$ which has passed phase I clinical trials due to its excellent anti-metastic activity [15].

A recent perspective article by Alessio et al, categorised metal anticancer compounds based on their mode of action, which could be divided into five different classes:

1. The metal has a functional role, i.e. the activity derives from a direct binding of the metal fragment to the biological target.

2. The metal has a structural role, it is instrumental in determining the shape of the compound and binding to the biological target occurs through non-covalent interactions.

3. The metal is a carrier for main ligands that are delivered in vivo. The metal might also protect the ligand before delivery. 
4. The metal compound behaves as a catalyst in vivo, e.g. through the production of reactive oxygen species (ROS) that cause cell damage.

5. The metal compound is photoactive and behaves as a photosensitizer.

Elucidation and optimisation of the mode of action of anticancer agents in classes 2, 4, and 5 as defined by Alessio et al require an intimate knowledge of both the structural and electronic properties of the complexes. Thus to fully realise the advantages of ruthenium complexes as novel inorganic therapeutics, it is crucial that the photophysical processes which govern the reactivity of the complexes are fully and comprehensively understood. Many research teams have incorporated 1,10-phenanthroline-5,6-dione (phendione) into the synthesis of a variety of ligands including Liu et al., who synthesised $p$-MOPIP with an OMe group ( $p=$ para position), $p$-HPIP with an OH group and $p$-NPIP with an NPIP group [16]. Liu et al also synthesised the corresponding ruthenium (II) complexes; $\left[\operatorname{Ru}(p h e n)_{2}(p-M O P I P)\right] 2+(1)$, where $\quad$-MOPIP is $\quad 2$-(4-methoxylphenyl)imidazo[4,5-f][1,10]phenanthroline, $\quad\left[\operatorname{Ru}(\mathrm{phen})_{2} \quad(p-\right.$ $\mathrm{HPIP})]^{2+}(\mathbf{2})$ where p-HPIP is 2-(4-hydroxyphenyl)imidazo[4,5-f][1,10]phenanthroline and $\left[\mathrm{Ru}(\mathrm{phen})_{2}\right.$ $(p-N P I P)]^{2+}(3)$ where p-NPIP is 2-(4-nitrophenyl)imidazo[4,5-f][1,10]phenanthroline [17]. The intrinsic binding constant, $\mathrm{k}_{\mathrm{b}}$ for the ruthenium complexes labelled 1,2 , and 3 are as follows; $0.20,0.69$, and 1.56 $\mathrm{x} 10^{5} \mathrm{M}^{-1}$, respectively. The order is in accordance with that of the electron withdrawing ability of the respective substituent $[-\mathrm{OR}<-\mathrm{OH}<-\mathrm{NPIP}]$. Such a trend in electronic effects of $\mathrm{Ru}(\mathrm{II})$ complexes binding to DNA can be reasonably explained by density functional theory (DFT) calculations [18].

This work serves to provide a link between the structural correlations observed in regarding biological activity and the electronic and excited state structure property relationships. Firstly the $\mathrm{Ru}(\mathrm{II})$ complexes will be thoroughly characterised though a systemic study of their electronic and vibrational properties.

Research into optimisation of electronic and emissive characteristics in organic polymeric and oligomeric systems has shown that the vibrational characteristics, and hence the non-radiative decay, can be gauged by both Stokes shift (crudely) and moreover routinely by Raman spectroscopy which 
probes the electron phonon coupling across the conjugated back-bone. Well-defined relationships relating the variation in conjugation across the $\pi$ back-bone to vibrational coherence have served to establish empirical relationships describing the variation of the fluorescence yield with Stokes shift, integrated Raman intensity and electronic band-gap [19, 20, 21, 22].

To this end, coordination compounds of $\mathrm{Ru}(\mathrm{II})$ were synthesised with the following nitrogen donor ligands; 2,2'-bipyrdine (bpy), 1,10-phenanthroline (phen) and 2,2'-biquinoline (biq). The step-by-step substitution of the auxiliary ligands allows analysis with essentially a single variable, increasing the reduction potential of the attached auxiliary ligand. The effect of the systematic change on the complexes properties is investigated using a series of ruthenium complexes with a end-group terminations varied from 2-(4-formylphenyl)imidazo[4,5-f][1,10] phenanthroline [FPIP], 2-(4cyanophenyl)imidazo[4,5-f][1,10] phenanthroline $[\mathrm{CPIP}]$, to 2-(4-nitrophenyl)imidazo[4,5-f][1,10] phenanthroline [NPIP].

It is proposed that the variation of the electronic band gap, extended to the Stokes shift, can be shown to have a well-defined dependence on the reduction potential of the attached auxiliary ligands, thus allowing the prediction of the band gap of further ruthenium based complexes. The sequential replacement of auxiliary ligands is anticipated to significantly influence the vibrational coupling along the complexes and as such the Raman spectroscopy is employed as a means to gauge the potential of vibrational coherence across the conjugated back-bone. This proposed change in vibrational coupling will allow for quantitative structure property relationships to be formed and any restriction of the avenues of non-radiative decay would lead to the maximizing the radiative decay, i.e., luminescence. Hence the study is aimed at exploration of structure-property relationships for both electronic and vibrational characteristics in these systems.

The establishment of well-defined structure property relationships, similar to the trend observed for the DNA binding mentioned earlier, will aid the intelligent design of complexes to absorb and emit in required spectral windows. Furthermore, the potential for control and optimisation of luminescence 
yield and lifetime is extremely desirable for applications such as photoactive therapeutics, solar cell dyes and oxygen sensor research where the excited state characteristics are extremely important, and elongation of the luminescence lifetime without compromising the yield is paramount.

\section{Experimental}

The ruthenium complexes investigated, shown in figure 1 , were $\left[R u(b p y){ }_{2} L\right]^{2+}$ and (b) $\left[R u(p h e n)_{2} L\right]^{2+}$ and (c) $\left[\mathrm{Ru}(\text { Biquin })_{2} \mathrm{~L}\right]^{2+}$ where $\mathrm{L}$ is the main ligand with differing functional group ligand terminations $(\mathrm{L}=$ 2-(4-formylphenyl)imidazo[4,5-f][1,10] phenanthroline (FPIP), 2-(4-nitrophenyl)imidazo[4,5$\mathrm{f}][1,10]$ phenanthroline (NPIP) and 2-(4-cyanophenyl)imidazo[4,5-f][1,10] phenanthroline (CPIP)..
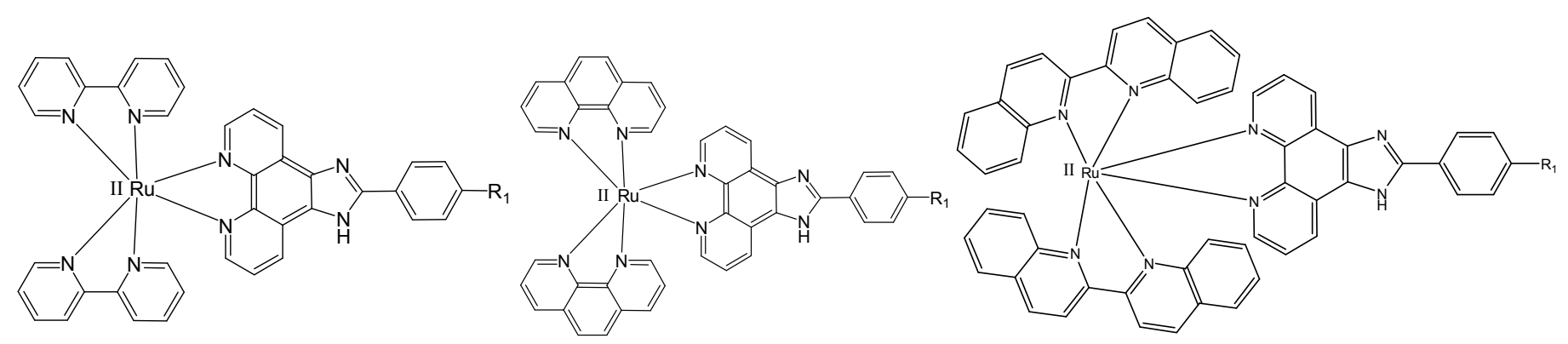

Figure 1. Ruthenium complexes, (a) $\left[\mathrm{Ru}(\mathrm{bpy})_{2} \mathrm{~L}\right]^{2+}$, (b) $\left[\mathrm{Ru}(\mathrm{phen})_{2} \mathrm{~L}\right]^{2+}$, and (c) $\left[\mathrm{Ru}(\mathrm{biq})_{2} \mathrm{~L}\right]^{2+}$ with $\mathrm{R}_{1}$ being $-\mathrm{CHO}, \mathrm{NO}_{2}$ and $\mathrm{CN}$ for the ligands (L) FPIP, NPIP and CPIP respectively

Synthesis of the ruthenium complexes, have been described elsewhere [18, 23, 24]. The complexes were prepared in an acetonitrile solution. Concentration dependent studies were undertaken to ensure the samples were unaffected by aggregation. Absorption spectroscopy was carried out using a Perkin Elmer Lambda 900 UV/VIS/NIR absorption spectrometer. The luminescence measurements were performed using a Perkin Elmer LS55 luminescence spectrometer. These measurements were used to calculate luminescence yields.

The reduction potential for the ligands is taken for literature and used as mean to describe the electronic state of the auxiliary ligand. The reduction potential can be defined as the tendency of a chemical 
species to acquire electrons and thereby be reduced. Hence it is a measure of the tenancy of the auxiliary ligands to contribute electrons across the $\mathrm{Ru}$ centre. It has been shown that the reduction potential of ruthenium complexes can be seen to vary in a regular and consistent manner with auxiliary ligand change and hence is an accurate parameter to use to establish the potential of electron donation [25]. Luminescence lifetimes of aerated samples were measured using a computer controlled Time Correlated Single Photon Counting Spectrometer FL900 from Edinburgh Instruments. A nanosecond nF900 flashlamp excitation source using deuterium gas at a pressure of $\sim 0.40$ bar provided the fluorescence excitation pulses at $300 \mathrm{~nm}$. A Peltier cooled Hamamatsu R955 side-window photomultiplier tube (PMT) was used in an orthogonal geometry. All decay curves were corrected using a deconvolution with the instrument response function obtained using a scattering solution. The profile of the instrument response pulse had a FWHM of $\sim 1$ ns which was the detection limit of the system. All samples were run using a variation in concentrations from $10^{-4} \mathrm{M}$ to $10^{-9} \mathrm{M}$ at $300 \mathrm{~K}$ and in all cases the lifetime was found to be concentration independent within $0.1 \mathrm{~ns}$.

Raman spectroscopy was performed using a Horiba Jobin-Yvon Labram HR800 UV confocal Raman imaging microscope system. A solid-sate $(785.1 \mathrm{~nm} / 3 \mathrm{~mW})$ laser source was used. The light was imaged to a diffraction-limited spot via the objective lens of an Olympus BX40 microscope. All experiments were carried out at room temperature $(300 \mathrm{~K})$. 
Results
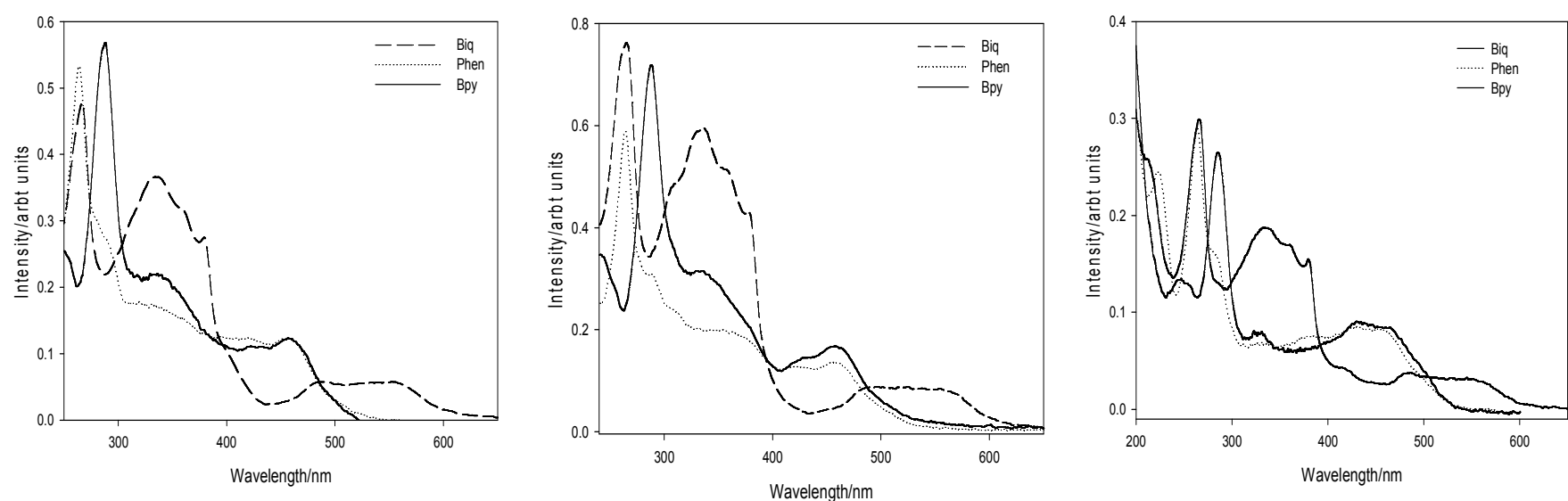

Figure 2. UV/Vis absorption spectra of ruthenium complexes, with R1 groups FPIP (a), CPIP (b) and NPIP (c)

The normalized absorption spectra of the ruthenium FPIP, CPIP and NPIP series can be seen in Figure 2a-c, respectively. The principle absorption of ruthenium complexes is made up of two main features at $\sim 300 \mathrm{~nm}$ and at $\sim 460 \mathrm{~nm}$ corresponding to the ligand centred (LC) absorption and a MLCT absorption, respectively $[26,27,28]$. Hence, an estimate of the energy of the corresponding singlet state $\left({ }^{1}\right.$ MLCT) can be made from the long-wavelength absorption band (Table 1) which for all complexes had a extinction co-efficient of $\sim 20,000 \mathrm{~L} \mathrm{~mol}^{-1} \mathrm{~cm}^{-1}$. It can be clearly seen that there is a systematic bathochromic shift of the important MLCT transition as the more electro-negative auxiliary ligands are attached. This shift is present in all complexes, regardless of the electronic nature of the main ligand. A more thorough description of the electronic changes by considering the FPIP, CPIP and NPIP series individually can be found below.

The most important absorption feature present is the MLCT transition and as such will be the focus of a detailed examination with regards to the systematic variation of the electronic nature of the auxiliary ligands. The first series to consider is the FPIP based complex with the absorption bands at $456 \mathrm{~nm}$ and $470 \mathrm{~nm}$ for the phenanthroline complex which combine to give the MLCT band absorption. On substitution of the phenanthroline (phen) with bipyridine (bpy), there is a small bathochromic shift in 
the MLCT band position. Further substitution with the larger, more conjugated, biquinoline (biq) ligand leads to a larger bathochromic shift of the MLCT absorption band to $550 \mathrm{~nm}$.

The second series is the CPIP terminated complexes which displayed the same substitution trends as observed in the previous complexes. The lowest wavelength absorption was found with the phenanthroline (phen) ligands attached, at $458 \mathrm{~nm}$ and $473 \mathrm{~nm}$, with a bathochromic shift to $460 \mathrm{~nm}$ and $481 \mathrm{~nm}$ incurred by bipyridine (bpy) substitution and further larger shift, to $551 \mathrm{~nm}$, displayed by the inclusion of the large biquinoline (biq) ligand.

The third series to be considered is the NPIP based complexes; again the phenanthroline (phen) auxiliary ligands show the longest wavelength absorption with peaks at $459 \mathrm{~nm}$ and $488 \mathrm{~nm}$. The introduction of the bipyridine (bpy) ligand produces a bathochromic shift of the absorption spectrum similar to that observed in the FPIP based complexes. The bathochromic shift is readily seen with the peak intensity features at $465 \mathrm{~nm}$ at $492 \mathrm{~nm}$. The introduction of the larger biquinoline (biq) ligand introduces a large bathochromic shift to an absorption peak at $553 \mathrm{~nm}$. It should be noted that the biquinoline (biq) based compounds showed a single absorption unlike the phenanthroline (phen) and bipyridine (bpy) counterparts. 
(a)

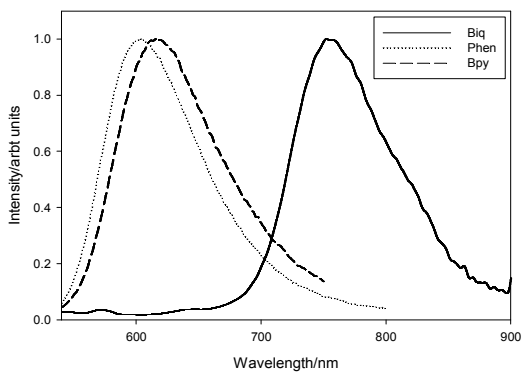

(b)

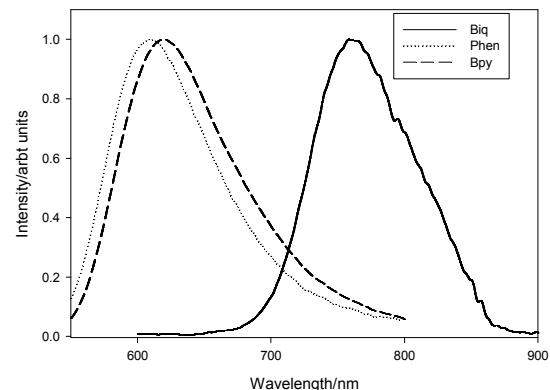

(c)

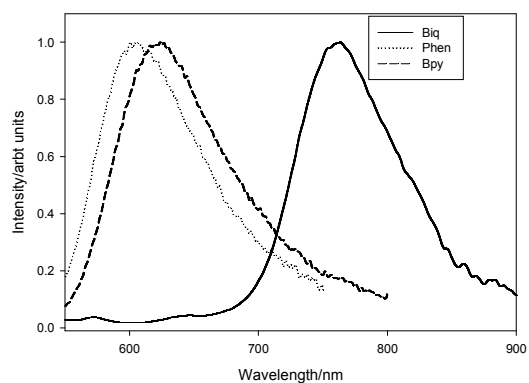

Figure 3. Normalised Luminescence spectra of ruthenium complexes, (a) $\left[\mathrm{Ru}\left(\mathrm{L}^{\prime}\right)_{2} \mathrm{FPIP}\right]^{2+}$, (b) $\left[\mathrm{Ru}\left(\mathrm{L}^{\prime}\right)_{2} \mathrm{CPIP}\right]^{2+}$, and (c) $\left[\mathrm{Ru}\left(\mathrm{L}^{\prime}\right)_{2} \mathrm{NPIP}\right]^{2+}$ where $\mathrm{L}^{\prime}$ is bpy, phen and biq respectively

The luminenesce spectra for all ruthenium complexes are shown in figure 3 and the emission peaks are tabulated in table 1. It can be clearly seen that there is a systematic bathochromic shift as the more electronegative auxiliary ligands are attached. This observed bathochromic shift mirrors the trend to longer wavelengths that was apparent in the absorption spectra. The luminescence spectra show far more prominent shifts than those observed in the absorption spectra. The FPIP based complexes show a luminescence peak at $589 \mathrm{~nm}, 598 \mathrm{~nm}, 750 \mathrm{~nm}$ for phenanthraline (phen), biprydine (bpy) and biquiniline (biq) respectively. The CPIP based compounds show the same progression trend, albeit with an increasing wavelength shift with the changing of the auxiliary ligands for phenanthraline (phen) to biquiniline (biq) via bpyridine (bpy). The increasing shift with change of auxiliary ligand is further manifested in the NPIP series, which has luminescence peak postions of $591 \mathrm{~nm}, 612 \mathrm{~nm}$, and $755 \mathrm{~nm}$ for the phenanthtroline (phen), bypridine (bpy), biquinoline (biq) compounds respectiviely.

Summing up the electronic transitions show a reduction in the excitation and emission energy of the comlexes with the changing of the auxiliary ligand from phenanthraline to bipyridine then biquiniline. This systematic tuning of the band-gap with the auxiliary ligands is most effective in the complexes with the greater electronegativity of the terminal functional group of the main ligand [29]. 
1

2

3

4

5

6

7

8

9

10

11

12

13

14
15

16

17

Table 1. Electronic data for ruthenium complexes aerated in acetonitrile at room temperature

\begin{tabular}{|c|c|c|c|c|c|c|}
\hline \multirow{2}{*}{} & \multicolumn{3}{|c|}{ UV/Vis $\boldsymbol{\lambda}_{\max } / \mathbf{n m}$} & \multicolumn{3}{c|}{ Luminescence $\boldsymbol{\lambda}_{\max } / \mathbf{n m}$} \\
\cline { 2 - 7 } & Bpy & Phen & Biq & Bpy & Phen & Biq \\
\hline FPIP & 460 & 456 & 550 & 598 & 589 & 750 \\
\hline NPIP & 466 & 463 & 553 & 612 & 591 & 755 \\
\hline CPIP & 460 & 458 & 551 & 606 & 590 & 752 \\
\hline
\end{tabular}

In order to account for the systematic result of the variation of the auxiliary ligands, the absorption and emission energies where plotted as a function of the reduction potential of the auxiliary ligand. 


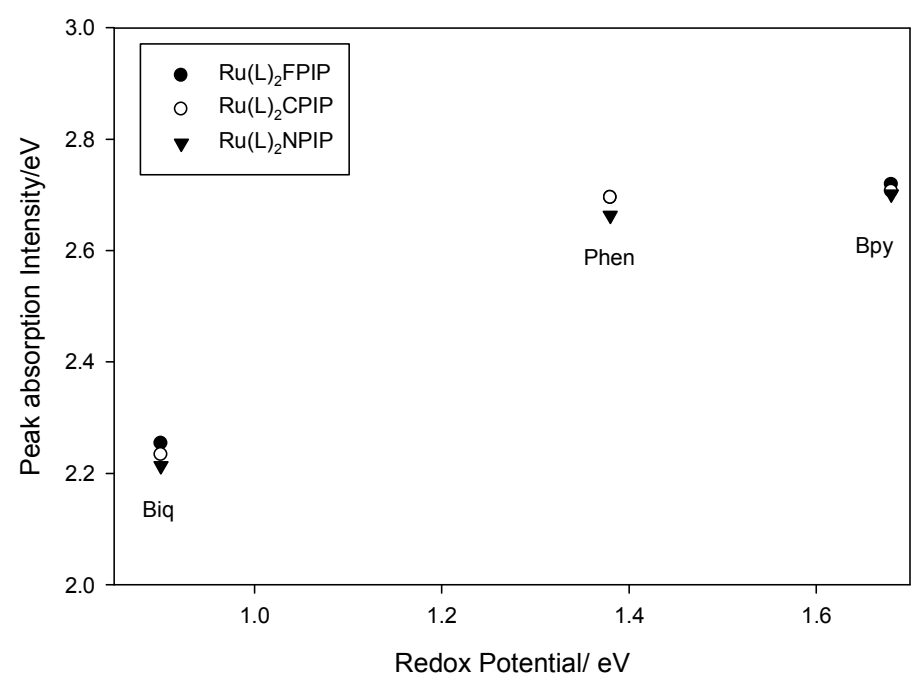

Figure 4. UV/Vis absorption characteristics against chemical potential $(\mu)$ of auxiliary ligand peaks

Figure 4 shows the variation of the position of the absorption maxima for the complexes plotted against the reduction potential of the auxiliary ligand as shown, by Magdesieva et al, [30]. It can be seen that a well-defined relationship exists between the MLCT transition energy and the electronic nature of the auxiliary ligand attached to the ruthenium centre. This defined variation in reduction potential matches the trend observed above and further reinforces our methodology of using an electronic term in order to quantify the electronic effect of the auxiliary ligand.

It can be clearly seen that the same well defined trend in the position of the peak absorption is evident for all complexes regardless of the main ligand end termination functional group. The largest variation with the systematic variation of the auxiliary ligand is for the FPIP series, closely followed by the CPIP and the NPIP series. The electron withdrawing functional group at the termination site will lead to the main ligand attracting electrons from the metal centre and hence acting to delocalise the electron density [31]. This delocalisation will lead to a larger effective conjugation length and hence a bathochromic shift for the absorption features [32]. The variation from a linear trend would suggest the influence of the terminal group as a means of moderating the effect of the auxiliary ligand change. It can be clearly seen form table 1 that the peak emission behaves in a similarly well-defined manner as shown by the absorption trends. 
The Stokes shift was taken as the difference between the peak absorption and the peak emission [33, 34, 35]. The FPIP terminated complexes show the smallest variation in Stokes shift with changing of auxiliary ligand, the NPIP showing the greatest variation with systematic change. It has been previously shown in conjugated organic systems that the Stokes shift can be seen as a crude measure of the nonradiative decay $[20,21,22]$ and hence a well defined variation of Stokes shift with luminescence yield would suggest the potential for tailoring the electronic and the vibrational (non-radiative decay) characteristics of the complexes. To further investigate the potential for such tailoring, the luminescence yield and lifetimes have been calculated as a means to elucidate the effect on the excited state lifetimes and yield.

Table 3. Luminescence data for ruthenium complexes aerated in acetonitrile

\begin{tabular}{|c|c|c|c|c|c|c|}
\hline \multirow{2}{*}{} & \multicolumn{3}{|c|}{ Luminescence lifetimes/ns } & \multicolumn{3}{c|}{ Luminescence Yield* } \\
\cline { 2 - 7 } & Bpy & Phen & Biq & Bpy & Phen & Biq \\
\hline FPIP & 138 & 103 & 4.6 & 0.037 & 0.030 & 0.016 \\
\hline CPIP & 125 & 107 & 3.2 & 0.039 & 0.032 & 0.018 \\
\hline NPIP & 72 & 33 & 2.7 & 0.012 & 0.0078 & 0.0030 \\
\hline
\end{tabular}

* Relative to 0.095 measured for $\left[\mathrm{Ru}(\mathrm{bpy})_{3}\right]^{2+}[36]$ 


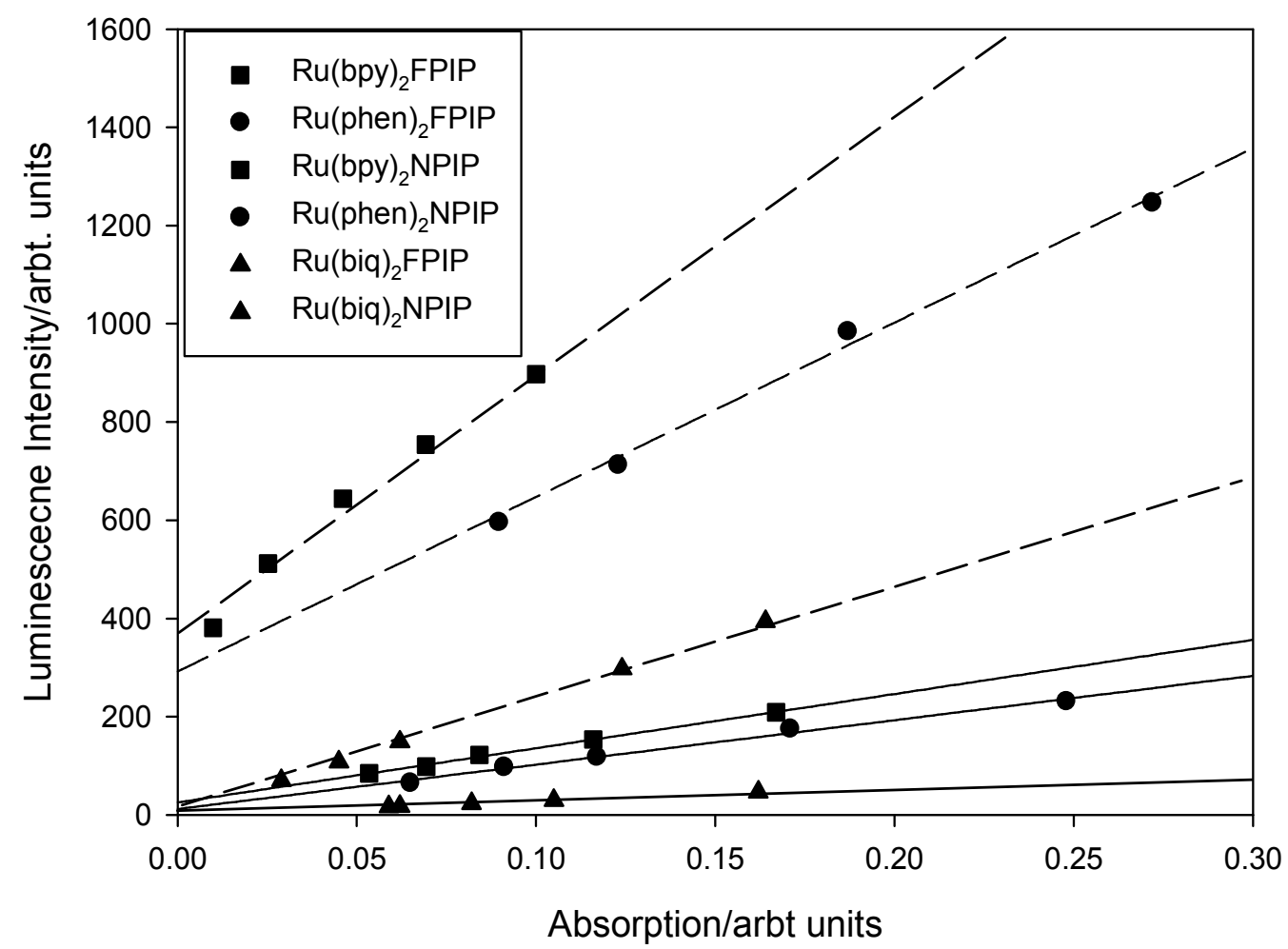

Figure 5. Luminescence intensity as a function of absorbance, slope indicates relative luminescence yield

Figure 5 shows the integrated relative luminescence yield of the nine complexes investigated. The values calculated for the yield are shown in table 3 . $\left[\mathrm{Ru}(\mathrm{bpy})_{3}\right]^{2+}$ was run as a standard and the luminescence yield results are relative to a literature value of 0.095 [36]. It can be readily seen that the bipyridine series shows the highest yields followed by the phenanthroline and then biquinoline. The contention that the Stokes shift is a measure of the non-radiative rate would lead to the assumption that the complexes with the smallest Stokes shift would have the greatest expected luminescence intensity, assuming the radiative rate being constant. 


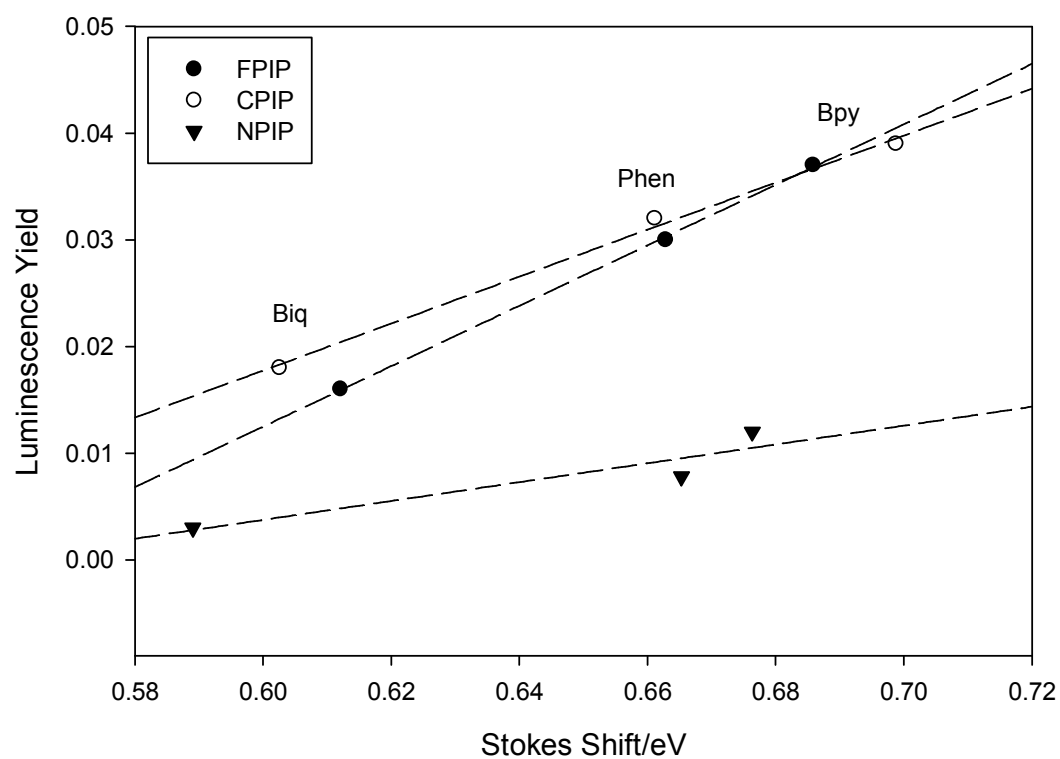

Figure 6. Luminescence yield versus peak Stokes shift for $\mathrm{Ru}(\mathrm{II})$ complexes, dotted lines are linear guide for the eye

To elucidate whether a well-defined relationship exists between the Stokes shift and luminescence yield, a graph of the FPIP, CPIP and NPIP series was plotted in Figure 6. From the graph, it is apparent that the Stokes shift is well-correlated with the luminescence yield. A linear fit to the data points is presented; the slope indicates the effect of the change of the auxiliary ligands goes from FPIP-CPIPNPIP. This variation slope mirrors the electronic nature as determined by the reduction potential and also the slope is well correlated with luminescence yield with the trend maintained as FPIP-CPIP-NPIP, going highest to lowest. The NPIP series show a greatly reduced slope indicating the effect of the change of the auxiliary ligands results in a reduction of the Stokes shift but with a concomitant reduction in the luminescence yield. This is counterintuitive to the theory that the vibrational and hence the non-radiative decay can be gauged by the Stokes shift, i.e. Stokes shift is inversely proportional to luminescence yield [21].

However this relationship is dependent on the conjugation length not being limited by the molecular size. If electron phonon coupling is restricted by molecular length of the complex, there are fewer 
vibrational modes over which to distribute energy, the excess energy per mode and thus the nuclear redistribution is amplified, increasing the Stokes shift [22]. It has been shown for oligomer-type systems that the Stokes shift can be seen to have proportional relationship with non-radiative decay rate [23]. As such the ruthenium complexes can be seen to behave akin to oligmeric structures wherein the conjugation is limited structurally rather than electronically. Hence the introduction of the systematically larger ringed systems increases the number of modes available for electron phonon coupling thus resulting in a reduced Stokes shift.

The changing slope of the linear fits would suggest that the electronic nature of the terminal group has a significant impact of the magnitude of the effect of systematically changing of the auxiliary ligand. This effect and merits further investigation and a complete study into the variation of the electronic and luminescence properties of the complex with variation of termination ligand type is currently being undertaken.

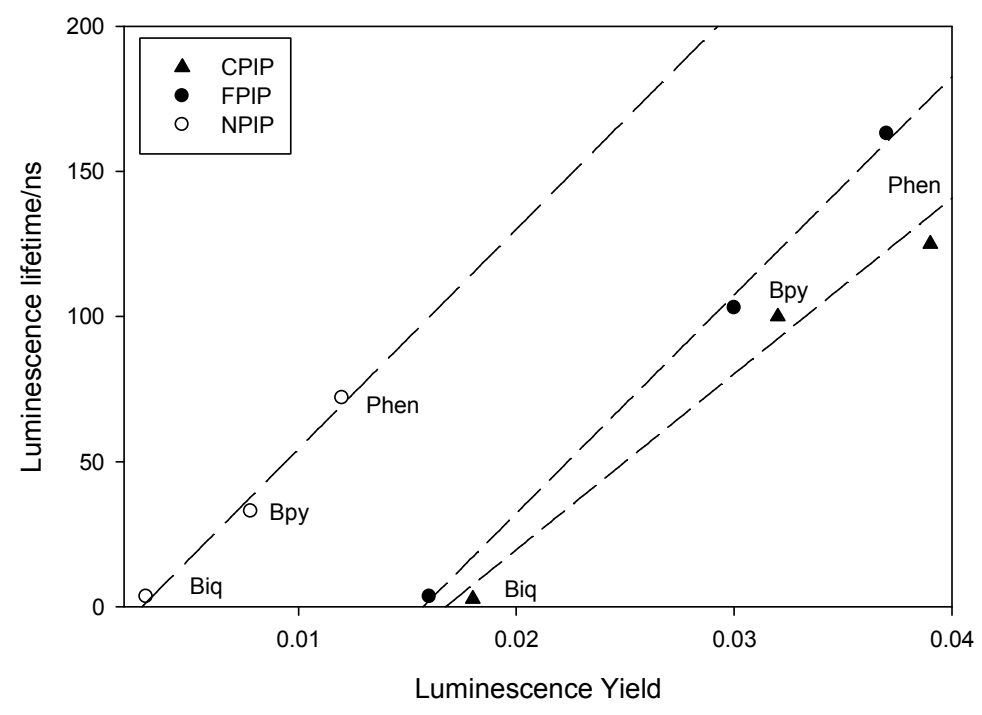

Figure 7. Luminescence lifetime against the luminescence yield, linear fit is a guide for the eye. 
In an effort to confirm that the non-radiative decay rate is indeed well defined, the luminescence lifetimes were then plotted against the relative luminescence yield, figure 7 . The luminescence yield was seen to increase as the Stokes shift was increased suggested the inverse relationship between the nonradiative rate and the Stoke shift. Thus a reduction of the non-radiative rate indicated by an increase in Stokes shift would lead to the longer lived excited state and thus a larger luminescence lifetime. The luminescence lifetime $\left(\tau_{1}\right)$ and the luminescence yield $\left(\Phi_{1}\right)$ can be described by the following equations [32]:

$$
\begin{aligned}
& \Phi_{\text {lum }}=\mathrm{k}_{\mathrm{rad}} /\left(\mathrm{k}_{\mathrm{rad}}+\mathrm{k}_{\mathrm{nrad}}\right) \\
& \tau_{\text {lum }}=\frac{1}{\left(\mathrm{k}_{\mathrm{rad}}+\mathrm{k}_{\mathrm{ic}}+\mathrm{k}_{\mathrm{isc}}\right)} \\
& \Phi_{\text {lum }}=\mathrm{k}_{\mathrm{rad}} \cdot \tau_{\text {lum }}
\end{aligned}
$$

Equation 2

Equation 3

Equation 4

Hence if the change in the radiative rate can be considered negligible, then a plot of the luminescence lifetimes against luminescence yield should yield a linear relationship, with a slope representative of the relative radiative rate. In Figure 7, this is shown to be the case for all series. This would suggest that the dominant factor being affected by the change of the auxiliary ligands is the non-radiative rate. The increase in the luminescence lifetime results in an almost linear increase in the relative yield. This suggests that the Stokes shift, as shown in figure 6 against luminescence yield, can be indicative of the magnitude of the non-radiative rate.

In order to further confirm that the Stokes shift can be used as a indirect guage of the non-radiative rate, the radiative $\left(\mathrm{k}_{\mathrm{rad}}\right)$ and non-radiative $\left(\mathrm{k}_{\mathrm{nrad}}\right)$ rate constants are calculated. Assuming that the intersystem crossing quantum yield for the formation of the ${ }^{3} \mathrm{MLCT}$ triplet state of our system is unity as for Ru (II) complexes and knowing the lifetimes $\left(\tau_{\text {lum }}\right)$ and emission quantum yields $\left(\Phi_{\text {lum }}\right)$, the radiative $\left(\mathrm{k}_{\mathrm{rad}}\right)$ and 
non-radiative $\left(\mathrm{k}_{\mathrm{nrad}}\right)$ rate constants for the FPIP, CPIP and NPIP series of complexes can be calculated using the following expressions:

$$
\begin{aligned}
& \mathrm{k}_{\mathrm{rad}}=\Phi_{\text {lum }} / \tau_{\text {lum }} \\
& \mathrm{k}_{\mathrm{nrad}}=\left(1-\Phi_{\text {lum }}\right) / \tau_{\text {lum }}
\end{aligned}
$$

\section{Equation 5}

Equation 6

Table 4. Aerated Photophysical Rates for Ruthenium polypyridyl complexes

\begin{tabular}{|c|c|c|c|c|c|c|}
\hline \multirow{2}{*}{} & \multicolumn{3}{|c|}{$\mathbf{k}_{\text {rad }} / \mathbf{s} \times 10^{5}$} & \multicolumn{3}{c|}{$\mathbf{k}_{\text {nrad }} / \mathbf{s} \times 10^{6}$} \\
\cline { 2 - 7 } & Bpy & Phen & Biq & Bpy & Phen & Biq \\
\hline FPIP & 2.68 & 2.91 & 34.8 & 6.97 & 9.42 & 213.9 \\
\hline CPIP & 3.12 & 2.99 & 56.3 & 7.69 & 9.05 & 306.9 \\
\hline NPIP & 1.67 & 2.36 & 11.1 & 13.9 & 30.6 & 369.3 \\
\hline
\end{tabular}
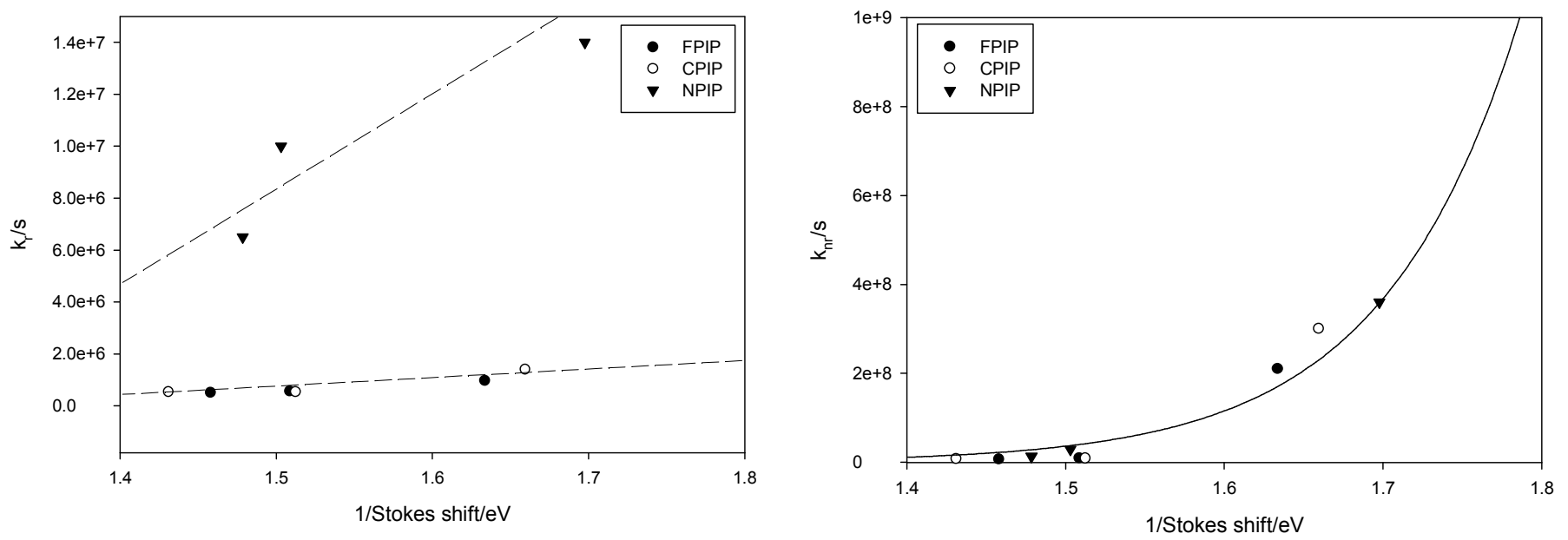

Figure 8. Inverse Stokes shift against (a) radiative and (b) non-radiative rate showing linear and exponential fit respectively. 
The graphs in figure 8 confirm the hypothesis that the photo-physical rate are well-defined, above the Stokes shift is shown to vary systemically with respect to both the radiative and non-radiative rate for all series. However it is apparent that the rate of variation of the radiative rate and the non-radiative rate as a function of the Stokes shift is different. The radiative rate shows a linear dependence with stokes shift where as the non-radiative rate is seen to vary exponentially with inverse Stokes shift. The exponential dependence seen here confirms the fact that the dominant process here is the change of nonradiative rate.

The Energy gap law, equating the increase in the non-radiative rate as the band-gap is reduced is a common empirical guide as to the excited state dynamic's of metal based complexes [37,38,39]. It has been shown that the Energy gap law can be written as

$$
k \mathrm{nr} \approx \exp \left(-\tilde{a} \Delta E / \hbar \grave{u ̀}_{\mathrm{m}}\right)
$$

\section{Equation 7}

where $\Delta E$ is the energy gap separation between the potential minima of the states involved, $\tilde{a}$ is a term that can be expressed in terms of molecular parameters, and $\grave{\mathrm{u}}_{\mathrm{m}}$ is the maximum and dominant vibrational frequency available in the system. It has been demonstrated that the non-radiative rate in metal based systems are governed by the Energy gap law, although deviation from a well-defined relationship are observed for low band-gap energies $<2.4 \mathrm{eV}$. Moreover, significant deviations from the predicted relationship are also observed [40,41], especially in the transition between monomeric and polymeric structures [42]. The non-radiative rate also shows a deviation from the predicted exponential relationships when dealing with complexes wherein the structure varies from a homologous complex.

With this in mind the Stokes shift, being a manifestation of both the electronic and vibrational characteristics of the complexes is shown to be a accurate gauge of the photophysical rates and is seen to vary in a well defined manner with regard to the radiative and non-radiative rate. The deviations observed in the Energy gap law correlations are absent when the non-radiative rate is plotted as a function of Stokes shift and a better exponential fit is obtained. Although the mechanism of non- 
radiative decay upon which the Energy gap law is based is intrinsically linked to the electron-lattice coupling of the material the Stokes shift having its origins in both the vibrational and electronic characteristics and is seen to be better able to account for the variation of non-radiative rate in proxy oligomeric systems where the conjugation is limited by structure rather than the electronic nature As is expected the non-radiative rates are dependent on both the electronic and vibrational coupling along the extended backbone of the metal based complexes. Thus, a vibrational study using Raman spectroscopy due to it sensitivity to the variation in the $\pi$ conjugation was employed to gauge the variation in a key vibrational mode along the conjugated back bone.

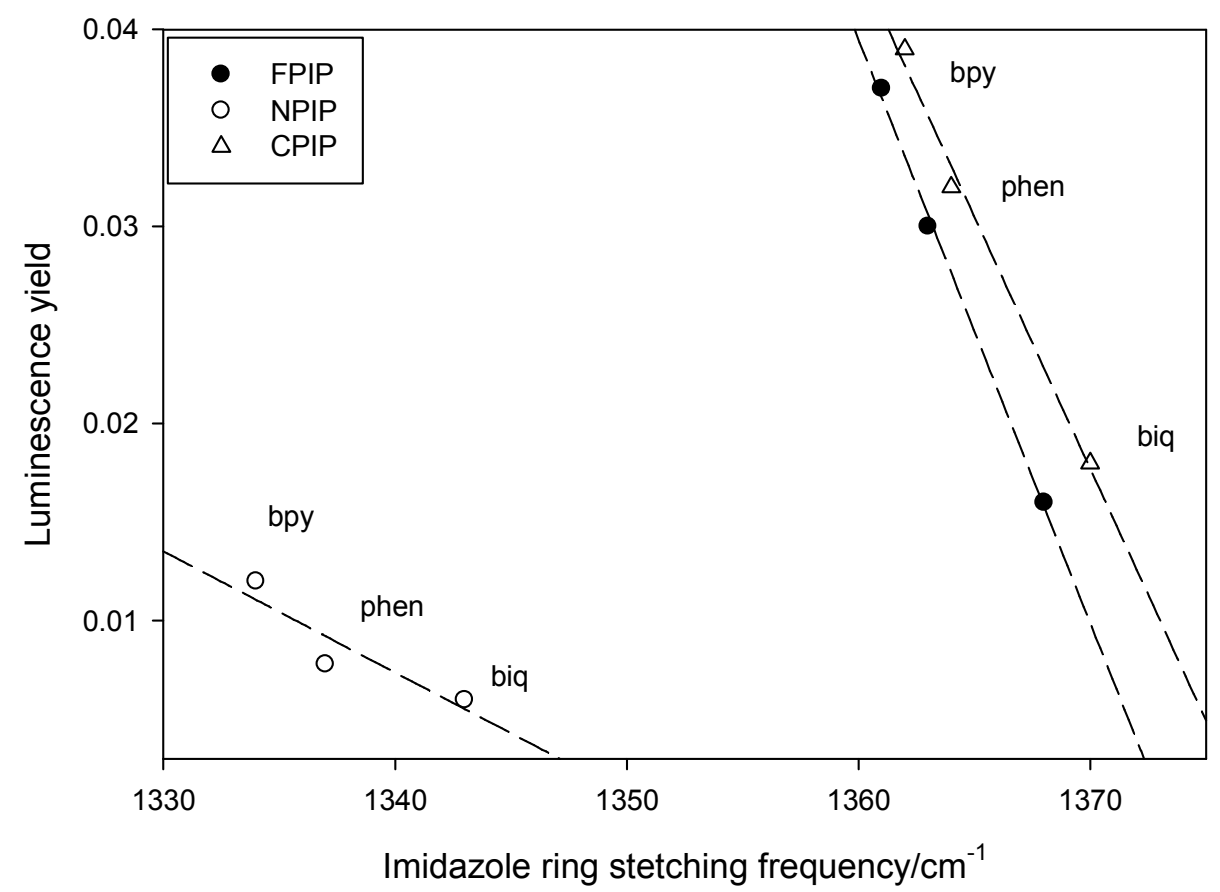

Figure 9. Imidazole ring stretching frequency against Luminescence yield.

Figure 9 shows the linear variation of the luminescence yield as a function of the variation of the frequency of the imidazole ring stretch of the main ligand. This vibrational mode was chosen as it is unique to the main ligand and conjugation and vibrational coherence across this bridging ring will determine the extent of the effective conjugation. The electronic nature of both the auxiliary ligands will 
considerably affect the electron density across the imidazole ring and therefore the vibrational frequency.

It is evident that the luminescence yield increases as the ring stretching frequency decreases. As seen previously for the electronic properties the FPIP and CPIP terminated complexes exhibit a similar activity profile. The NPIP series shows a linear behaviour but with significantly reduced slope.

This linear behaviour can be reconciled with the electronic effects of the auxiliary as the variation of the electron density proceeds from biquinoline to the sequentially less eectron donated phenanthroline and then to bipyridine. The electron density close to the $\mathrm{Ru}$ centre limits the strength of the $\pi$ conjugation extending across the main ligand; hence with the end group electronic potential remaining constant, the effective delocalisation is a function of the electron density of the attached auxiliary ligands.

However the change in the slope of the linear fit can be directly related to the change of the end group terminations of the main ligand. The greater the electron withdrawing potential of the end group, the smaller the slope. Hence the slope inclination can be found to follow the trend, NPIP $\rightarrow$ CPIP $\rightarrow$ FPIP. The electron withdrawing effect of the end group reduces the electron density on the imidazole ring thus reducing the stretching frequency. This effect will be dealt with in subsequent paper where the electronic nature of the terminal groups and their effect will be explored.

In summary the effect of the increasing electron density of the auxiliary ligands increases the electronic conjugation which has been shown to decrease the luminescence by strengthening the vibrational coherence across the imidazole ring and thus the potential for non-radiative decay. Once the main ligand is constant the effect of auxiliary ligand change is relatively easy to reconcile, however the changing of both main and auxiliary ligand will introduce competing effects. These effects make a unified expression of the effect of the auxiliary/main ligand change difficult to quantify, but it is apparent that there are both vibrational and electronic components to this effect. Further work will be undertaken to elucidate the effect of the variation of the terminal end group electronegative on the photo-physical rates and an empirical formulae to reconcile the push-pull effects simultaneous auxiliary and main ligand change. 


\section{Conclusions}

This study establishes structure property relationships for a multiple series of systematically varied ruthenium complexes through a thorough investigation of the electronic absorption and emission characteristics. A well-defined relationship is found to exist between the absorption and emission wavelengths and the electronic effect of auxiliary ligand as measured by the electronic chemical potential. The relative electron withdrawing/donating effects of the auxiliary ligands are shown to be the dominant factor in tailoring the electronic characteristics of the complexes but it is shown that this effect can be moderated by the electron affinity of the terminal functional group on the main ligand when investigating the luminescence lifetime/yield. The electron withdrawing ligands terminating the complex work to negate the magnitude of the effect of changing the auxiliary ligands.

As has been previously shown, for conjugated organic systems [21,22], the Stokes shift as defined herein can be used as a crude measure of vibrational activity and hence the non-radiative decay rate and is seen to be well-defined with respect to the luminescence yield of the complexes. This relationship is complex due to the effective conjugation being limited by molecular length rather than inherent electronic properties. The relationship between the luminescence yield and lifetime establishes that the dominant effect of the auxiliary ligands being systematically change is a manipulation in the nonradiative decay rate. The dominance of the changing non-radiative rate is further illustrated as a function of Stokes shift and illustrates the potential for the Stokes shift to be a more accurate gauge of nonradiative rate change than the Energy gap law as it has its origin in both the electronic re-organisation and the vibrational characteristics of the complexes. Raman spectroscopy of the complexes leads to the assignment of a vibrational mode unique to the main ligand, the frequency of which is shown to vary in a well-defined manner with the luminescence yield, indicating it could be a used as an empirical guide to the push-pull effect of auxiliary ligand change. These important relationships allow the tailored extension of the luminescence lifetime without the loss of luminescence yield. This is highly desirable 
in therapeutics where the mode of action is based on the photoactive behaviour or for the production of reactive oxygen species (ROS) that cause cell damage.

\section{Acknowledgements}

The FOCAS Institute is funded under the National Development Plan 2000-2006 with assistance from the European Regional Development Fund. L. Perdisatt acknowledges the Abbest Scholarship support.

\section{References}

1. Florea, A-M.; Büsselberg, D; Cancer, 2011, 3, 1351-1371;

2. Siddik, Z. H.; Oncogene, 2003, 22, 7265-7279

3. Ozols, R.F.; Cancer Treat. Rev., 1991, 18, 77-83.

4. Roberts, J.J., Pera, J.J. Jr.; Am. Chem. Soc., 1983, 3-25

5. Fricker, S.P.; Dalton Trans., 2007, 1-15

6. Heffeter, P.; Jungwirth, U. ; Jakupec, M.; Hartinger, C.; Galanski, M.; Elbling, L.; Micksche, M.; Keppler, B.; Berger. W.; Drug Resist. Updates, 2008, 11, 1-16

7. Kostova, I. ; Curr. Med. Chem., 2006, 13, 1085-1107

8. Gianferrara, T., Bratsos, I. , Alessio, E. ; Dalton Trans., 2009, 7588- 7598

9. Nguyen, H. M. ; Nguyen, D. N.; Kim, N.; Adv. Na.l Sci.: Nanosci and Nanotech., 2010, 1, 025001

10. Payne, S. J., Fiore, G. L., Fraserand, C. L., Demas, J. N. ; Anal. Chem., 2010, 82, 3, 917-921

11. Rose, M. J., Mascharak, P. K. ; Coord. Chem. Rev., 2008, 252, 2093-2114

12. Sava, G. , Pacor, S., Zorzet, S., Alessio, E. , Mestroni, G. ; Pharm. Res., 1989, 21, 5, 617-628

13. Clarke, M. J., Zhu, F. , Frasca, D. R., Chem. Rev. 1999, 99, 2511; Clarke, M. J., 
Coord. Chem. Rev. 2003, 236, 209

14. 'Metal Complexes in Cancer Chemotherapy', Ed. B. K. Keppler,VCH: Weinheim

(Germany)Mestroni, G. , Alessio, E. , Sava, G. Pacor, S. , Coluccia, M. , , 1993, 157;

15. Gianferrara, T., Bratsos, I. , Alessio, E. ,; Dalton Trans, 2009, 7588- 7598

16. Liu, J., Huang J-W, Fu, B., Zhao, P. , Yu, H-C. , Ji, L-N.; Spectroscheimi Acta A, 2007, 67, 2, 391-394

17. Shi, S. , Liu, J. , Li, J. , Zheng, K.C, Tan, C.P, Chen, L.M, Ji, L.; Dalton Trans., 2005, 11, 20382046

18. Liu, J., Mei, W.J., Lin, L.J., Zheng, K.C., Chao, H., Yun, F.C. , Ji, L.N. , Inorg Chim. Acta, $2004357285-293$

19. O’Neill, L., Lynch, P. , McNamara, M. , Byrne, H. J. ; Polymer, 2008, 49, 4109-4114;

20. Photophysics of Organic Conjugated Systems, L. O’Neill, 2006, Dublin Institute of Technology

21. O’Neill, L., Byrne, H. J. ; J. Phys. Chem. B, 2005, 109, 12685-12690

22. O’Neill, L., Lynch, P. , McNamara, M. , Byrne, H. J. ; J. Phys. Chem. B, 2007, 111, 7999-8005

23. Perdisatt, L., O’Neill, L. , O’Connor, C.; Polyhedron, submitted 2012

24. Chao, H. , Li, R-H., Jiang, C-W., Li, H. , Ji, L-N., Li X-Y.; J Chem. Soc., Dalton Trans., 2001, 1920-1926,

25. Wu, F., Thummel, R.P., Inorganica Chimica Acta, 2002, 327, 26-30

26. Physical Chemistry by A.G. Whittaker, A.R. Mount \& M.R. Heal, 2000

27. Messori, L., Abbate, F., Marcon, G. , Orioli, P. , Fontani, M. , Mini, E., Mazzei, T. , Carotti, S., O’Connell, T. , Zanello, P. ; J. Med. Chem., 2000, 43, 19, 3541.

28. Hager, G.D., Crosby, G.A. ; J. Am. Chem.Soc. 1975, 97,7031-7037

29. Organic Chemistry by J. Clayden, N. Geeves, S. Warren, Oxford, $2^{\text {nd }}$ Edition, 2012

30. Magdesieva, T.V., Butin, K.P. ; Russ. Chem. Revs., 1993, 62, 4, 359-385

31. Herrero-García, N., del Rosario Colorado Heras, M., del Rosario Torres, M., Fernández, I., Osío Barcina, J.; Euro J. Org Chem., 2012, 13, 2643-2655. 
32. Sauteret, C., Hermann, J.P., Frey, R., Pradere, F., Ducuing, J., Baughman, R.H., Chance, R.R. ; Phys. Rev. Lett. 1976, 36, 956.

33. Organic Molecular Photophysics by J.B Birks, John Wiley and Sons, 1973

34. Endicott, J. F. , Schlegel, H. B. Jamal Uddin, Md., Seniveratne, D. S. ; Coord. Chem. Revs., 2002, 229, 95-106.

35. Bräm, O. , Messina, F. , El-Zohry, A. M. , Cannizzo, A. , Chergui, M. ; Chem. Phys., 2012, 393, $51-57$.

36. Suzuki, K., Kobayashi, A., Kaneko, S., Takehira, K., Yoshihara, T., Ishida, H., Shiina, Y., Oishi, S., Tobita, S., Phys Chem. Chem. Phys., 2009,11, 9850-9860

37. Principles of Fluorescence Spectroscopy, Vol 1 by Joseph R. Lakowicz, 2006

38. Arias, M. , Concepcion, J., Crivelli, I. , Delgadillo, A. , Diaz, R. , Francois, A. , Gajardo, Lopez, R., Leiva, A. M., Loeb, B. , Chem. Phys., 2006, 326, 1, 54-70.

39. Ross, H. B., Boldaji, M., Rillema, D. P , Blanton, C. B., White, R. P., Inorg. Chem., 1989, $28,6,1013-1021$

40. Chen, H-L., Huang, Y-F., Hsu, C.-P. , Lim, T-S., Kuo, L-C. , Leung, M-K. Chao, T-C, Wong, K-T., Chen, S-A, Fann, W. ; J. Phys. Chem. A, 2007, 111, 9424-9430

41. Latouche, C., Lanoë, P-H., Williams, J. A. G., Guerchais, V., Boucekkine, A., JFillaut, -L. ; New J. Chem.., 2011, 35, 2196-2202

42. Wilson, J. S., Chawdhury, N. , Al-Mandhary, M. R. A., Younus, M., Khan, M. S., Raithby, P. R., Kolhler, A., Friend, R. H. , J. Am. Chem. Soc., 2001, 123, 9412-9417 


\section{ToC graphic}

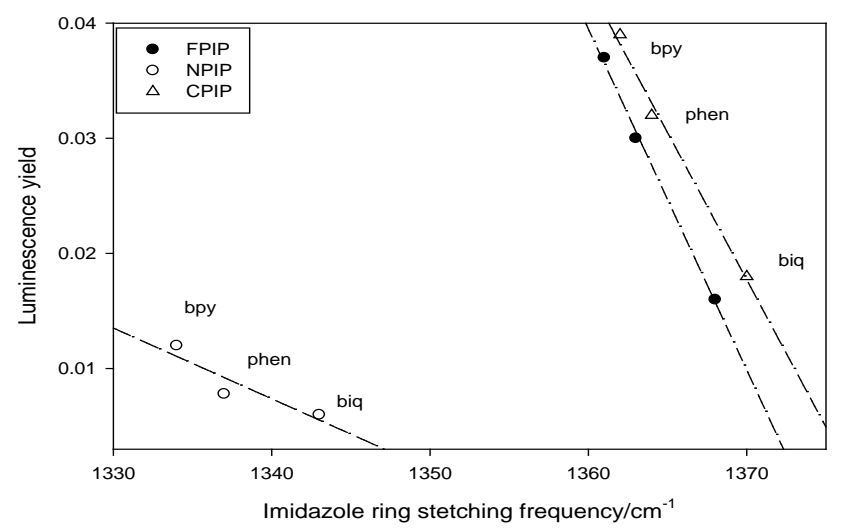

ToC :- Imidazole ring stretching frequency against Luminescence yield. 